\title{
Transferring elements of a density matrix
}

\author{
Armen E. Allahverdyan ${ }^{1)}$ and Karen V. Hovhannisyan ${ }^{1,2)}$ \\ 1) Yerevan Physics Institute, Alikhanian Brothers Street 2, Yerevan 375036, Armenia \\ 2) Yerevan State University, A. Manoogian Street 1, Yerevan, Armenia
}

\begin{abstract}
We study restrictions imposed by quantum mechanics on the process of matrix elements transfer. This problem is at the core of quantum measurements and state transfer. Given two systems A and $\mathrm{B}$ with initial density matrices $\lambda$ and $r$, respectively, we consider interactions that lead to transferring certain matrix elements of unknown $\lambda$ into those of the final state $\widetilde{r}$ of $\mathrm{B}$. We find that this process eliminates the memory on the transferred (or certain other) matrix elements from the final state of A. If one diagonal matrix element is transferred, $\widetilde{r}_{a a}=\lambda_{a a}$, the memory on each nondiagonal element $\lambda_{a \neq b}$ is completely eliminated from the final density operator of A. Consider the following three quantities $\Re \lambda_{a \neq b}, \Im \lambda_{a \neq b}$ and $\lambda_{a a}-\lambda_{b b}$ (the real and imaginary part of a non-diagonal element and the corresponding difference between diagonal elements). Transferring one of them, e.g., $\Re \widetilde{r}_{a \neq b}=\Re \lambda_{a \neq b}$, erases the memory on two others from the final state of A. Generalization of these set-ups to a finite-accuracy transfer brings in a trade-off between the accuracy and the amount of preserved memory. This trade-off is expressed via system-independent uncertainty relations which account for local aspects of the accuracy-disturbance trade-off in quantum measurements.
\end{abstract}

PACS numbers: 03.65.-w, 03.67.-a

\section{INTRODUCTION}

Quantum mechanics imposes constraints on information processing. Among known examples of such contraints is the the fact that measuring an unknown quantum state inevitably disturbs it. This fundamental feature was known since the early days of quantum mechanics [1], and has been recently formalized via uncertainty relations and information-disturbance trade-offs [2-7]; see [8, 9] for reviews.

Another constraint is the no-cloning theorem, which states that due to linearity and unitarity of quantum dynamics there exists no physical process that can produce perfect copies of a system that is initially in an unknown quantum state [10]. The theorem is closely related to the quantum measurement induced state-disturbance [11]. There are several important generalizations of the no-cloning theorem [12-22].

Here we study limitations imposed by quantum mechanics on the process of matrix elements transfer from one system to another. This problem includes as particular cases quantum measurement and cloning (see below for details). Before we formally pose the problem in the next section, let us see where such transfer processes are encountered.

\section{A. Quantum measurement}

Let a quantum system is prepared in a (generally, unknown) state described by a density matrix $\rho_{\mathrm{S}}$. For the measurement of an observable $\hat{A}$ pertaining to the system quantum theory predicts that the probabilities of observing various eigenvalues of $\hat{A}$ are given by the Born rule:

$$
\operatorname{Pr}(\hat{A}=a) \equiv \operatorname{tr}\left[\rho_{\mathrm{S}} \hat{\Pi}_{\hat{A}}(a)\right]
$$

where $\hat{\Pi}_{\hat{A}}(a)$ is the projector referring to the eigenvalue $a$ of $\hat{A}$.

For describing the measurement process one has to include explicitly the measuring apparatus, which-prior to its interaction with the system - is in a known state with a density matrix $\rho_{\mathrm{M}}$. Several requirements on $\rho_{\mathrm{M}}$ and the system-apparatus interaction are to be satisfied by an ideal quantum measurement [26]. The basic - and in a sense minimal - requirement is that the initial probabilities $\operatorname{Pr}(\hat{A}=a)$ in (1) are mapped to the final probabilities of the apparatus observable $\hat{B}[3,4,23,25,26]$ :

$$
\operatorname{Pr}(\hat{A}=a)=\operatorname{Pr}_{\text {fin }}(\hat{B}=a) \equiv \operatorname{tr}\left[\rho_{\mathrm{M}}^{\mathrm{fin}} \hat{\Pi}_{\hat{B}}(a)\right],
$$

where $\rho_{\mathrm{M}}^{\mathrm{fin}}$ is the final (after interacting with the system) density matrix of the apparatus, $\hat{\Pi}_{\hat{B}}(a)$ is the projector of $\hat{B}$, and where for simplicity we assumed that $\hat{A}$ and $\hat{B}$ have the same discrete spectra.

Eq. (2) implies that the probabilities (1) of $\hat{A}$ can be obtained by looking at the statistics of the apparatus observable $\hat{B}$. Relation (2) is satisfied with many models of ideal quantum measurements [25-27]. It is supposed to hold for an arbitrary initial density matrix $\rho_{\mathrm{S}}$, because the latter is unknown.

Thus the quantum measurement means, in particular, transferring the initial matrix elements of the tested system in the representation where $\hat{A}$ is diagonal. The full transfer amounts to requiring (2) for all independent probabilities. However, for concrete purposes we can be interested only by certain probabilities $\operatorname{Pr}_{\text {in }}(\hat{A}=a)$ and then (2) is to be imposed only for those probabilities.

For many models of quantum measurements it was observed that after realizing an ideal measurements of the observable $\hat{A}$, the system is left in a state with a density matrix diagonal in the $\hat{A}$-representation [25-27]. This feature is closely related to the von Neumann projection [25-27]. It is now interesting to ask what does happen 
to the state of the system after transferring ideally the diagonal matrix elements according to (2), i.e., after satisfying the minimal condition of quantum measurements.

\section{B. Polarization transfer}

Transfer of matrix elements is realized also in one of the main methods of cooling, where polarization is transferred from one system to another [28-30], e.g., from highly polarized electron spins to almost unpolarized nuclear spins [30]. Polarization transfer is well known in NMR/ESR, quantum/atomic optics, semiconductor physics, etc [28-30]. For the simplest example take two spin- $\frac{1}{2}$ density matrices for two systems

$$
\lambda=\frac{1}{2}[1+\vec{l} \vec{\sigma}], \quad r=\frac{1}{2}[1+\vec{r} \vec{\sigma}],
$$

where $\vec{\sigma}$ are Pauli matrices, and $\vec{l}, \vec{r}$ are Bloch vectors. Transferring diagonal (non-diagonal) elements $\lambda_{11}=\widetilde{r}_{11}$ $\left(\lambda_{12}=\widetilde{r}_{12}\right)$ amounts to transferring the $z(x$ and $y)$ component(s) of the Bloch vectors. Both these processes are well-studied experimentally [28-30,35]. Related processes of energy (excitation) transfer are important in biological systems (e.g., photosynthesis) [31]. The energy transfer between two quantum system means transferring the diagonal elements in the energy representation.

\section{State transfer}

Quantum communication via (unknown) state transfer plays an important role both for practical implementation of scalable quantum processors and for understanding the efficiency of quantum computation; see $[32,33]$ for reviews. In many theoretical studies devoted to the state-transfer problem one simply assumes that the state of a finite-dimensional quantum system (qubits or qutrits) is transferred to another system. Qubits and qutrits can be understood literally as real systems with a finite number of energy levels. However, more often than not, finite-dimensional system are implemented in subspaces of a larger dimensional quantum system; see [34] for a review. For instance, qubits can be implemented via bosonic modes, or alternatively, they can be placed in subspaces of a multi-qubit system, the purpose being immunization of the qubit from decoherence (decoherencefree subspaces) of feasibility of error-correcting schemes [34].

Without going into details of implementation of qubits and qutrits in a larger dimensional systems (see [34] in this context) one can state that in all those cases where qubits and qutrits are not understood literally, the resulting quantum state can be described via suitable matrix elements of the full density matrix of the larger (embodying) system; see [35] for experimental realizations. Thus, in all those cases transferring quantum state refers to certain (not all) elements of the full density matrix.
For various schemes of quantum state transfer it is of a clear interest to understand what happens to the state of the source system after the transfer has been realized, e.g., to what extent this final state can serve as a source for another state transfer?

These examples show that transferring (certain) elements of the (unknown) density matrix and understanding limitations imposed by quantum theory on such processes is a relevant task.

The paper is organized as follows. We formally state the studied problem in section II. The next two sections discuss limitation related to the ideal transfer of matrix elements. In particular, section III discusses how the obtained results related to quantum measurements. Section $\mathrm{V}$ describes a set-up for non-ideal transfer processes. Details of such processes are presented in sections VI and VII. We summarize in the last section.

\section{STATEMENT OF THE PROBLEM}

Consider a finite-dimensional quantum system A. The information is encoded into matrix elements of its density operator $\lambda$; this situation is realized in the above examples. To be a carrier of information this state has to be unknown. For simplicity we assume that the state is completely unknown.

There is another, composite system $\mathrm{B}+\mathrm{C}$ in some known state with density operator $\omega$. The Hilbert spaces of $\mathrm{A}$ and $\mathrm{B}$ have the same dimension: $\operatorname{dim} \mathcal{H}_{\mathrm{A}}=$ $\operatorname{dim} \mathcal{H}_{\mathrm{B}}=N$. The initial state of the overall system $\mathrm{A}+\mathrm{B}+\mathrm{C}$ is $\lambda \otimes \omega$. Let $p, r=1, \ldots, N$ and

$$
\{|p\rangle\}_{p=1}^{n},\langle p \mid r\rangle=\delta_{p r}, \quad\{|\bar{p}\rangle\}_{p=1}^{n},\langle\bar{p} \mid \bar{r}\rangle=\delta_{p r},
$$

be two orthonormal bases in $\mathcal{H}_{\mathrm{A}}$ and $\mathcal{H}_{\mathrm{B}}$, respectively. The interaction between $\mathrm{A}$ and $\mathrm{B}+\mathrm{C}$ is described by unitary operator $U$. It will be chosen such that for any initial density operator $\lambda$ of A, certain initial matrix elements $\lambda_{a b}=\langle a|\lambda| b\rangle$ of

$$
\lambda=\sum_{p r} \lambda_{p r}|p\rangle\langle r|
$$

are equal to the corresponding matrix elements of the final state $\widetilde{r}$ of $\mathrm{B}$ :

$$
\lambda_{a b}=\widetilde{r}_{a b}=\langle\bar{a}|\widetilde{r}| \bar{b}\rangle, \quad \widetilde{r}=\operatorname{tr}_{\mathrm{A}+\mathrm{C}}\left(U \lambda \otimes \omega U^{\dagger}\right) .
$$

Here $\mathrm{C}$ is an auxiliary system (ancilla or environment). After tracing it out, the considered dynamic operation amounts to a trace-preserving completely positive map acting on $\mathrm{A}+\mathrm{B}$.

We aim to understand implications of the matrix elements transfer from A to B on the memory of the transferred elements $\lambda_{a b}$ (or some other elements of $\lambda$ ) in the final state $\widetilde{\lambda}=\operatorname{tr}_{\mathrm{B}+\mathrm{C}}\left(U \lambda \otimes \omega U^{\dagger}\right)$ of $\mathrm{A}$ (the formal definition of memory is given in section $\mathrm{V}$ ).

Note that when all density matrix elements are transferred, the final state of A cannot be equal to its initial state. This follows from the no-cloning theorem: 
there exists no quantum process that can produce perfect copies of a system that is initially in an unknown quantum state [10]. The theorem is closely tied to the fact that measuring the unknown quantum state inevitably disturbs it [11]. However, the no-cloning principle - even in the form of its various generalizations [12-20] - cannot be applied directly to our problem, since here only certain (not all) matrix elements are copied (transferred).

We choose the initial state of $\mathrm{B}+\mathrm{C}$ as

$$
\omega=|\overline{1}\rangle\langle\overline{1}|\otimes| C\rangle\langle C|,
$$

where $|C\rangle$ lives in the Hilbert space $\mathcal{H}_{\mathrm{C}}$ of $\mathrm{C}$. This choice does not restrict generality provided that there are no restrictions on the dimensionality of the Hilbert space $\mathcal{H}_{\mathrm{C}}$ of $\mathrm{C}$, and provided that we are free to design unitary evolutions for $\mathrm{B}+\mathrm{C}$. Indeed, an initial mixed state of $\mathrm{B}+\mathrm{C}$ can be purified by extending $\mathrm{C}$ to a larger Hilbert space, while the resulting pure state can be rotated to $|\overline{1}\rangle \otimes|C\rangle$ by a suitable unitary operator.

We represent the unitary operator $U$ as $(p=1, \ldots, N)$

$$
U|p\rangle \otimes|\overline{1}\rangle \otimes|C\rangle=\sum_{k, l}|k\rangle \otimes|\bar{l}\rangle \otimes\left|C_{k l}^{p}\right\rangle \equiv\left|\psi_{p}\right\rangle,(5)
$$

where all summation indices run from 1 to $N$, and where the vectors $\left|C_{k l}^{p}\right\rangle$ with $p, k, l=1, \ldots, N$ live in $\mathcal{H}_{\mathrm{C}}$.

The unitarity of $U$ amounts to $(p, r=1, \ldots, N)$

$$
\left\langle\psi_{p} \mid \psi_{r}\right\rangle=\delta_{r p} \quad \text { or } \quad \sum_{k l}\left\langle C_{k l}^{p} \mid C_{k l}^{r}\right\rangle=\delta_{r p} .
$$

The final states

$$
\tilde{\lambda} \text { and } \quad \widetilde{r}=\sum_{a, b} \widetilde{r}_{a b}|\bar{a}\rangle\langle\bar{b}|
$$

of $\mathrm{A}$ and $\mathrm{B}$, respectively, read from (5)

$$
\begin{aligned}
& \tilde{\lambda}=\sum_{p r} \lambda_{p r} \Theta_{p r}, \\
& \widetilde{r}_{a b}=\sum_{p r} \lambda_{p r} \sum_{k}\left\langle C_{k b}^{r} \mid C_{k a}^{p}\right\rangle,
\end{aligned}
$$

where

$$
\Theta_{p r} \equiv \sum_{k n}|k\rangle\langle n| \sum_{l}\left\langle C_{n l}^{r} \mid C_{k l}^{p}\right\rangle .
$$

The process of matrix elements transfer depends crucially on which (diagonal or non-diagonal) elements are transferred. We therefore study these cases separately. Note that a diagonal density matrix $\lambda$ (with unknown diagonal matrix elements) carries only a classical information. Non-diagonal elements represent quantum aspects of the information contained in the unknown state $\lambda$.

\section{DIAGONAL TO DIAGONAL TRANSFER: THE IDEAL SITUATION}

Assume that for every initial state $\lambda$ of $\mathrm{A}$ a diagonal element $\lambda_{a a}$ of $\mathrm{A}$ is transferred to the diagonal element $\widetilde{r}_{a a}$ of B:

$$
\lambda_{a a}=\widetilde{r}_{a a} .
$$

For this it is necessary to have [see (8)]

$$
\sum_{k}\left\langle C_{k a}^{r} \mid C_{k a}^{p}\right\rangle=\delta_{p r} \delta_{p a} \text { for all pairs }(r, p) .
$$

Eq. (11) for $r=p=a$ implies $\sum_{k}\left\langle C_{k a}^{a} \mid C_{k a}^{a}\right\rangle=1$. Combining this with (6) under the same condition $p=r=a$ gives $\left|C_{k l}^{a}\right\rangle=0$ for $l \neq a$. Eq. (11) for $r=p=c \neq a$ gives $\left|C_{k a}^{c}\right\rangle=0$ for every $c \neq a$. Altogether, we get

$$
\begin{gathered}
\sum_{l}\left\langle C_{n l}^{a} \mid C_{k l}^{c}\right\rangle=0 \text { for every } c \neq a \text { or } \\
\Theta_{a \neq c}=0,
\end{gathered}
$$

implying from $(7,9)$ that due to transferring $\lambda_{a a}=\widetilde{r}_{a a}$ the memory on each initial non-diagonal element $\lambda_{a \neq c}$ in the final density operator $\widetilde{\lambda}$ of $\mathrm{A}$ is lost; see (7).

Let us stress that the final state $\tilde{\lambda}$ of A need not be diagonal and that the memory on $\lambda_{a a}$ itself is conserved in $\widetilde{\lambda}$. Note that to be able to speak on the memory and its loss, we have to have initially some freedom in choosing $\lambda_{a \neq c}$, i.e., the latter should carry some information.

Recall from our introductory discussion that transferring the diagonal elements is an essential part of the quantum measurement. The above result on the memory loss of non-diagonal elements shows in which specific sense the state of the measured system is disturbed after the measurement. Studying disturbances induced by various quantum measurement - in particular, studying the inevitable disturbance as a function of the measurement accuracy — is a known subject; see [5-9] for reviews. In particular, the analysis of various models for the quantum measurement led to a conclusion that after the ideal measurement is completed, the post-measurement state is diagonal (an effect sometimes attributed to decoherence) $[26,27]$. It is seen from $(7,9)$ and from (10-12) that after the ideal transfer of all diagonal matrix elements the final state of A need not be diagonal, though it looses the memory on all non-diagonal elements of the initial state of A. Indeed, assuming that all diagonal are transferred we get from $(10,12)$ for the final state $\widetilde{\lambda}$ of A:

$$
\langle s|\widetilde{\lambda}| t\rangle=\sum_{p} \lambda_{p p}\left\langle C_{t p}^{p} \mid C_{s p}^{p}\right\rangle .
$$

This means that the diagonalization of the postmeasurement state was a consequence of various additional conditions imposed on the quantum measurement process; see [26] for a detailed discussion.

To repeat, the basic (and minimal) requirement for the quantum measurement is the transfer of diagonal matrix elements, and this requirement leads to elemination of memory rather than to diagonalization.

\section{TRANSFER OF NON-DIAGONAL ELEMENTS.}

Demanding

$\sum_{k}\left\langle C_{k b}^{r} \mid C_{k a}^{p}\right\rangle=\delta_{r b} \delta_{p a}$ for all $(r, p)$ and $a \neq b$, 
amounts to transferring ideally the corresponding nondiagonal element:

$$
\widetilde{r}_{a b}=\lambda_{a b}
$$

for arbitrary initial state $\lambda$ of $\mathrm{A}$; see $(7,9)$. The nonnegativity of $\sum_{k}\left[\alpha^{*}\left\langle C_{k a}^{a}\left|+\beta^{*}\left\langle C_{k b}^{b}\right|\right]\left[\alpha\left|C_{k a}^{a}\right\rangle+\beta\left|C_{k b}^{b}\right\rangle\right]\right.\right.$ as a function of two complex numbers $\alpha$ and $\beta$ (CauchySchwartz inequality) leads to

$$
1=\sum_{k}\left\langle C_{k a}^{a} \mid C_{k b}^{b}\right\rangle \leq \sqrt{\sum_{k}\left\langle C_{k a}^{a} \mid C_{k a}^{a}\right\rangle \sum_{k}\left\langle C_{k b}^{b} \mid C_{k b}^{b}\right\rangle},
$$

where the equality in (14) is due to (13) under $r=a$ and $k=b$. The inequality in (14) has to be saturated, since (6) implies $\sum_{k}\left\langle C_{k a}^{a} \mid C_{k a}^{a}\right\rangle \leq 1, \sum_{k}\left\langle C_{k b}^{b} \mid C_{k b}^{b}\right\rangle \leq$ 1. Thus we have $\sum_{k}\left\langle C_{k a}^{a} \mid C_{k a}^{a}\right\rangle=\sum_{k}\left\langle C_{k b}^{b} \mid C_{k b}^{b}\right\rangle=1$, which together with (6) gives for any $k$

$$
\left|C_{k l}^{a}\right\rangle=0 \text { for } l \neq a \text { and }\left|C_{k l}^{b}\right\rangle=0 \text { for } l \neq b .
$$

Eqs. $(7,9,15)$ lead to $\Theta_{a \neq b}=\Theta_{b \neq a}=0$, i.e., the memory on the transferred non-diagonal element $\lambda_{a b}$ in the final density operator $\widetilde{\lambda}$ is lost; see (7).

Another consequence of saturating the inequality in (14) is that $\left|C_{k b}^{b}\right\rangle=\left|C_{k a}^{a}\right\rangle$ for any $k$, which leads to

$\sum_{l}\left\langle C_{n l}^{a} \mid C_{k l}^{a}\right\rangle=\left\langle C_{n a}^{a} \mid C_{k a}^{a}\right\rangle=\sum_{l}\left\langle C_{n l}^{b} \mid C_{k l}^{b}\right\rangle=\left\langle C_{n b}^{b} \mid C_{k b}^{b}\right\rangle$,

i.e., $\Theta_{a a}=\Theta_{b b}$, meaning that memory on the difference of diagonal elements $\lambda_{a a}-\lambda_{b b}$ in the final density operator $\widetilde{\lambda}$ is lost; see (7). Thus one ideal nondiagonal-tonondiagonal transfer eliminates the memory on three real quantities, while one diagonal-to-diagonal ideal transfer eliminates memory on $2(N-1)$ real quantities. The difference between these two cases is that for the ideal nondiagonal-to-nondiagonal transfer the memory on the transferred element itself is eliminated from the final state of A. This means that the non-diagonal elements (as compared to diagonal ones) carry a different [more fragile] type of information.

Let us note that when only the real part of the nondiagonal element is transferred, $\Re \widetilde{r}_{a b}=\Re \lambda_{a b}$, for any initial density matrix $\lambda$ of $\mathrm{A}$, the above result on elimination of the memory on $\lambda_{a a}-\lambda_{b b}$ still holds, while only the memory on the imaginary part $\Im \lambda_{a b}$ is eliminated from the final density operator $\widetilde{\lambda}$ of $\mathrm{A}$ (and vice versa when transferring the imaginary part $\left.\Im \widetilde{r}_{a b}=\Im \lambda_{a b}\right)$. Likewise, transferring the difference between the eigenvalues, $\widetilde{r}_{a a}-\widetilde{r}_{b b}=\lambda_{a a}-\lambda_{b b}$, eliminates the memory on $\Im \lambda_{a b}$ and on $\Re \lambda_{a b}$. The derivation of these facts is similar to that given around (13-15). In this sense these three quantities $\lambda_{a a}-\lambda_{b b}, \Im \lambda_{a b}$ and $\Re \lambda_{a b}$ are complementary to each other.

It is seen that transferring an eigenvalue $\widetilde{r}_{a a}=\lambda_{a a}$ implies different (more severe) consequences for the memory of non-diagonal elements, than transferring an eigenvalaue difference $\lambda_{a a}-\lambda_{b b}$. Nevertheless, when all $N-1$ independent diagonal elements are transferred either directly, or via their differences, the resulting damage to the memory of non-diagonal elements is the same, i.e., the memory on all non-diagonal elements is erased. For the direct transfer this is obvious from (12), while for the second situation of transferring the eigenvalue differences this follows from the fact that $\widetilde{r}_{a a}-\widetilde{r}_{b b}=\lambda_{a a}-\lambda_{b b}$ implies conditions (15).

\section{NON-IDEAL TRANSFER AND A MEASURE OF MEMORY}

While the above results refer to the ideal transfer, it is important to see how much memory can be preserved under a non-ideal, finite-accuracy transfer. Naturally, the general purpose of studying non-ideal transfer is to find some compromise between transferring diagonal elemenens and erasing the memory of non-diagonal elements in the final state of $\mathrm{A}$.

First let us recall an obvious fact that when transferring (ideally or not) diagonal elements (i.e., positive numbers summing to one), we have to describe the transfer of independent diagonal elements only.

Now if the ideal transfer corresponds to $\widetilde{r}_{a a}^{[\mathrm{id}]}=\lambda_{a a}$, its non-accurate version is defined to be

$$
\widetilde{r}_{a a}=\varepsilon_{a} \lambda_{a a}
$$

where we assume that $\varepsilon_{a}$ does not depend on the initial state $\lambda$, and where $\frac{\widetilde{r}_{a a}^{[\mathrm{id}]}-\widetilde{r}_{a a}}{\widetilde{r}_{a a}^{\text {[id] }}}=1-\varepsilon_{a}$ varies between zero and one, $0<1-\varepsilon<1$, and characterizes the relative accuracy of the transfer. (Clearly, one cannot have $\varepsilon_{a}>1$, because the positive diagonal elements should sum to one for all initial state $\lambda$; we also recall that (16) is demanded for independent probabilities only.) The notion of the relative accuracy is frequently met in the standard analysis of experimental errors [36].

If $\lambda_{a a}$ is considered as a signal, $\varepsilon_{a}<1$ corresponds to reducing (by a fixed amount) the signal magnitude without introducing any bias. If some noise is present during the actual transfer of the matrix element, this reduction will correspond to decreasing the signal-to-noise ratio, because weaker signals are more difficult to detect [36].

Conditions (16) are to be imposed on independent probabilities only, so that at best we can have only $N-1$ such constraints.

Note that (16) is certainly not the only way of defining non-ideal measurements. For instance, in the literature devoted to quantum measurements one sometimes employs the Heisenberg representation [4, 24]. Within this representation there is a reasonable definition of non-ideality, which is related to considering Heisenberg operators as signals [4, 24]. In particular, the Heisenberg operator of the apparatus variable after the systemapparatus interaction is compared to the system-variable Heisenberg operator before this interaction [4]. Other 
approaches to non-ideal measurements are reviewed in [7-9].

However, condition (16) seems to be the simplest possibility (at least within the employed Schroedinger representation) for introducing a finite non-accuracy without introducing any bias.

\section{A. Quantifying the memory}

The memory on the initial non-diagonal element $\lambda_{a \neq c}$ in the final state (7) is most naturally quantified by checking the response of the final state to perturbations in $\lambda_{a \neq c}$. We take another initial state $\lambda^{\prime}$ of $\mathrm{A}$, such that all matrix elements of $\lambda$ and $\lambda^{\prime}$ are identical besides the real and/or imaginary part of $\lambda_{a \neq c}$. Naturally, such a $\lambda^{\prime}$ can always be found, due to the basic constraint on $\lambda_{a \neq c}:\left|\lambda_{a \neq c}\right| \leq \lambda_{a a} \lambda_{c c}$. (If $\lambda_{a a}=0$ (or $\lambda_{c c}=0$ ), the very freedom in choosing $\lambda_{a \neq c}$ is absent, so there is no point in discussing its memory loss.)

Provided that the (small) difference between $\lambda$ and $\lambda^{\prime}$ is fixed, we look at the difference between the corresponding final states $\widetilde{\lambda}$ and $\widetilde{\lambda}^{\prime}$. This amounts to taking the derivatives $\partial \tilde{\lambda} /\left.\partial \Re \lambda_{a c}\right|_{\Im \lambda_{a c}}$ and $\partial \tilde{\lambda} /\left.\partial \Im \lambda_{a c}\right|_{\Re \lambda_{a c}}$, which quantify, respectively, the memory on the real and imaginal parts of $\lambda_{a c}$. These are still matrices, but the strength of the dependence of $\tilde{\lambda}$ on $\Re \lambda_{a \neq c}$ or on $\Im \lambda_{a \neq c}$ can be characterized via norms $\left\|\partial \widetilde{\lambda} /\left.\partial \Re \lambda_{a c}\right|_{\Im \lambda_{a c}}\right\|$ and $\left\|\partial \tilde{\lambda} /\left.\partial \Im \lambda_{a c}\right|_{\Re \lambda_{a c}}\right\|$. Since all norms are equivalent in a finite-dimensional Hilbert space -i.e., given two norms $\|.\|_{1}$ and $\|.\|_{2}$, there exist positive constants $a$ and $b$ such that $a\|A\|_{2} \leq\|A\|_{1} \leq b\|A\|_{2}$ for any matrix $A$-we work with the Euclidean norm

$$
\|A\| \equiv \sqrt{\operatorname{tr}\left(A A^{\dagger}\right)}
$$

where $A^{\dagger}$ is the hermitean conjugate of $A$. Finally, the memory of $\widetilde{\lambda}$ on $\lambda_{a \neq c}$ (i.e., on both $\Re \lambda_{a \neq c}$ and $\Im \lambda_{a \neq c}$ ) is defined as

$$
\frac{1}{2} \sqrt{\left\|\partial \widetilde{\lambda} / \partial \Re \lambda_{a c}\right\|^{2}+\left\|\partial \widetilde{\lambda} / \partial \Im \lambda_{a c}\right\|^{2}}=\left\|\Theta_{a \neq c}\right\|,
$$

where $\frac{1}{2}$ is introduced for convenience, and where $\Theta_{a c}$ is defined in (9).

That the memory of $\widetilde{\lambda}$ on (the real and imaginary parts of) $\lambda_{a c}$ can be characterized by $\left\|\Theta_{a c}\right\|$ is verified also by studying the matrix gradient of $\widetilde{\lambda}$, whose modulus is limited by $\left\|\Theta_{a \neq c}\right\|$ and $\frac{1}{\sqrt{2}}\left\|\Theta_{a \neq c}\right\|$ from above and below, respectively [38].

Note that in the initial state $\left\|\Theta_{a c}\right\|=1$ (perfect memory), while after a trace-preserving completely positive map $\lambda \rightarrow \widetilde{\lambda}$, we get that the memory on a matrix element can only decrease $\left\|\Theta_{a c}\right\| \leq 1$. We skip the derivation of this fact, because it is very similar to the derivation presented around (23-25). Now assume that after transferring matrix elements, when $\mathrm{A}$ has reached the state $\tilde{\lambda}$, the system $\mathrm{A}$ is subjected to a closed-system dynamics: $\tilde{\lambda} \rightarrow \hat{U} \tilde{\lambda} \hat{U}^{\dagger}$, where $\hat{U}$ is a unitary operator living in the Hilbert space of $\mathrm{A}$, and generated by the free Hamiltonian of A. Physically, this means that there is time-lag between realizing the matrix elements transfer and checking for memory. Now as follows from the unitary invariance of the norm (17), $\|A\|=\left\|\hat{U} A \hat{U}^{\dagger}\right\|$, the memory on a non-diagonal matrix element will not change under a local (closed-system) dynamics.

It is thus seen that the introduced measure of memory does have desired features that support its interpretation. The above reasoning can be applied to quantifying the memory on various combinations of matrix elements; see below.

\section{Fidelity}

Note that for describing the state disturbance during quantum measurements and cloning one frequently employs the fidelity between the final and initial state; see, e.g., $[8,9,15]$. For our situation this implies that for quantifying disturbances in the state of $\mathrm{A}$, we try to use the fidelity $F(\lambda, \widetilde{\lambda})$ between the initial $\lambda$ and final $\widetilde{\lambda}$ states of A:

$$
F(\lambda, \widetilde{\lambda})=\left(\operatorname{tr} \sqrt{\left[\lambda^{1 / 2} \widetilde{\lambda} \lambda^{1 / 2}\right]}\right)^{2} .
$$

Features of the fidelity are reviewed in [37]. In particular, $F(\lambda, \widetilde{\lambda})$ varies between 0 and 1 and it is equal to 1 if and only if $\lambda=\widetilde{\lambda}$. Thus, its deviation from 1 is supposed to quantify the "distance" between $\lambda$ and $\widetilde{\lambda}$. The largest "distance" $F(\lambda, \widetilde{\lambda})=0$ is achieved for orthogonal states $\lambda$ and $\tilde{\lambda}$.

We saw above that the memory on non-diagonal elements disappeared after the diagonal elements transfer. This naturally means that the final state of A differs from its initial state, and thus the fidelity is smaller than one. The converse is clearly not correct: the fidelity strictly smaller than one yet does not imply the specific memory loss effect found above. In other words, for the present problem the global measures of the state disturbance (such as the fidelity) are not adequate, because they can hide important physics. We need a local description of the disturbances induced in the final state of the source system A, such as the measure of memory introduced above.

Looking at the situation from a different angle, let us note the following undesirable feature of the fidelity (as would-be employed for the present situation). At the end of section V A we noted that the introduced measure of memory is invariant with respect to unitary (closedsystem) dynamics. This is clearly not the case with the fidelity, because in general $F(\lambda, \widetilde{\lambda}) \neq F\left(\lambda, \hat{U} \widetilde{\lambda} \hat{U}^{\dagger}\right)$ for a unitary $\hat{U}$. We note in this context that a clear analysis 
of various general drawbacks of the fidelity is presented in Ref. [21].

\section{DIAGONAL TO DIAGONAL TRANSFER: NON-IDEAL SITUATION.}

We shall study the maximal possible memory on the initial non-diagonal elements $\lambda_{a \neq c}$ under a finiteaccuracy transfer (16). It proves more convenient to assume $N \geq 3$ and to start immediately with the simultaneous non-ideal transfer of two (independent) diagonal elements of the $N \times N$ density matrix:

$$
\widetilde{r}_{a a}=\varepsilon_{a} \lambda_{a a}, \quad \widetilde{r}_{b b}=\varepsilon_{b} \lambda_{b b}, \quad 0<\varepsilon_{a}<1, \quad 0<\varepsilon_{b}<1,
$$

where $\varepsilon_{a}$ and $\varepsilon_{b}$ do not depend on the initial state $\lambda$ and quantify the non-ideality. This case is generic, since the non-ideal transfer of one (or several) elements can be recovered from it; see below. (For $N=2$ we have only $\widetilde{r}_{a a}=\varepsilon_{a} \lambda_{a a}$ instead of (19).) Instead of (11) we get from (19)

$$
\sum_{k}\left\langle C_{k u}^{r} \mid C_{k u}^{p}\right\rangle=\varepsilon_{u} \delta_{p r} \delta_{p u} \text { for all }(r, p) \text { and } u=a, b .
$$

Eq. (20) for $r=p \neq a$ and for $r=p \neq b$ gives for any $k$

$$
\left|C_{k a}^{p}\right\rangle=0 \text { for } p \neq a \text { and }\left|C_{k b}^{p}\right\rangle=0 \text { for } p \neq b .
$$

Given $(19,20,21)$ we now establish an upper bound on $\left\|\Theta_{a \neq c}\right\|$. Let us define

$$
z_{n l k l}^{r p} \equiv\left\langle C_{n l}^{r} \mid C_{k l}^{p}\right\rangle,
$$

and let $\sum_{l}^{\prime}$ be the summation over $l=1, \ldots, N$ excluding $l=a$ and $l=b$. We get from $(7,9)$

$$
\begin{aligned}
\left\|\Theta_{a \neq c}\right\|^{2} \equiv \sum_{k, n} \mid & \left.\sum_{l} z_{n l k l}^{c a}\right|^{2} \leq \sum_{k, n}\left[\sum_{l}\left|z_{n l k l}^{c a}\right|\right]^{2} \\
& \leq \sum_{k, n}\left[\sum_{l}^{\prime} \sqrt{z_{n l n l}^{c} c} \sqrt{z_{k l k l}^{a} a}\right]^{2} \\
& \leq \sum_{n} \sum_{l}^{\prime} z_{n l n l}^{c} \sum_{k} \sum_{l}^{\prime} z_{k l k l}^{a a}
\end{aligned}
$$

where the inequalities in (24) and (25) are due to the Cauchy-Schwartz inequality, while in (24) we additionally used (21). We now get from $(25)$ and $(6,20,21)$

$$
\begin{aligned}
& \left\|\Theta_{a \neq b}\right\| \leq \sqrt{\left(1-\varepsilon_{a}\right)\left(1-\varepsilon_{b}\right)}, \\
& \left\|\Theta_{a \neq c}\right\| \leq \sqrt{\left(1-\varepsilon_{a}\right)} \text { for every } c \neq a, c \neq b . \\
& \left\|\Theta_{b \neq c}\right\| \leq \sqrt{\left(1-\varepsilon_{b}\right)} \text { for every } c \neq a, c \neq b .
\end{aligned}
$$

These inequalities - which are akin to the uncertainty relations - relate the non-ideality of transfer to the maximal possible amount of the conserved memory. Note that the bound on $\left\|\Theta_{a \neq b}\right\|$ is tighter than those on $\left\|\Theta_{a \neq c}\right\|$ and $\left\|\Theta_{b \neq c}\right\|:$ once the diagonal elements $\lambda_{a a}$ and $\lambda_{b b}$ are transferred, the memory o the cross-non-diagonal element $\lambda_{a b}$ is the most vulnerable one.

The extension of $(26,27)$ to transferring non-ideally several matrix elements should be obvious, since the nondiagonal elements under such a transfer fall naturally into two classes, which correspond to $(26)$ and $(27,28)$ respectively.

Let us show that the bounds $(26,27)$ are saturated by the proper choice of $\left|C_{k n}^{p}\right\rangle$. To this end assume that $\operatorname{dim} \mathcal{H}_{\mathrm{C}}=1:\left|C_{k b}^{p}\right\rangle=C_{k b}^{p}|C\rangle$, where $C_{k b}^{p}$ are c-numbers satisfying (6). Thus we study a unitary interaction between $\mathrm{A}$ and $\mathrm{B}$. Choosing for $N=3$

$$
\begin{gathered}
C_{11}^{1}=\sqrt{\varepsilon_{1}}, \quad C_{13}^{1}=\sqrt{1-\varepsilon_{1}}, \quad C_{22}^{2}=\sqrt{\varepsilon_{2}}, \\
C_{23}^{2}=\sqrt{1-\varepsilon_{2}}, \quad C_{33}^{3}=1
\end{gathered}
$$

while all other $C_{k b}^{p}$ with $p, k, b=1,2,3$ are zero, we satisfy the unitarity conditions (6) and realize the optimal memory-conserving non-ideal transfer (19) with $a=1$ and $b=2$. Now $(26,27)$ become equalities.

\section{NON-IDEAL TRANSFER OF NON-DIAGONAL ELEMENTS}

Let us now turn to a finite-accuracy, non-diagonal-tonon-diagonal transfer

$$
\widetilde{r}_{a b}=\eta \lambda_{a b}, \quad a \neq b \quad \text { and } \quad 0<|\eta|<1,
$$

where $\eta$ can be a complex number, and where $|\eta|$ characterizes the accuracy in the same sense as $\varepsilon_{a}$ in (16). We shall find out how the memory in the non-diagonal element $\left\|\Theta_{a b}\right\|$ and the memory $\left\|\Theta_{a a}-\Theta_{b b}\right\|$ on the difference betwen the diagonal elements are bounded. Initially, we restrict ourselves to finding the maximal possible memories for the c-number case

$$
\left|C_{k b}^{p}\right\rangle=C_{k b}^{p}|C\rangle .
$$

Already this particular case will allow us to draw general conclusions on the difference with the non-ideal diagonalto-diagonal transfer. More general cases will be discussed below.

For (31) to hold for an arbitrary initial state $\lambda$ of A we need

$$
\sum_{k} C_{k b}^{r} C_{k a}^{p *}=\eta \delta_{r b} \delta_{p a} \text { for all }(r, p) \text { and } a \neq b .
$$

This implies $\sum_{k} C_{k b}^{a} C_{k a}^{a *}=\sum_{k} C_{k b}^{b} C_{k a}^{b *}=0$, and then

$$
\begin{gathered}
|| \Theta_{a \neq b} \|^{2}=\phi_{a}^{a} \phi_{a}^{b}+\phi_{b}^{a} \phi_{b}^{b}+\Lambda_{a b}, \\
\phi_{v}^{u} \equiv \sum_{k}\left|C_{k v}^{u}\right|^{2}, \\
\Lambda_{a \neq b} \equiv \sum_{[s l]}^{\prime}\left[\sum_{k} C_{k l}^{a} C_{k s}^{a *}\right]\left[\sum_{n} C_{n s}^{b} C_{n l}^{b *}\right],
\end{gathered}
$$

where $\sum_{[s l]}^{\prime}$ means that the four pairs $(s, l)=$ $(a, a),(a, b),(b, a),(b, b)$ are excluded from the summation 
over $s=1, \ldots, N$ and $l=1, \ldots, N$. In estimating $\left|\Lambda_{a \neq b}\right|$ from above we proceed by applying the Cauchy-Schwartz inequality and using (34):

$$
\begin{array}{r}
\left|\Lambda_{a \neq b}\right| \leq \sum_{[s l]}^{\prime}\left[\sum_{k}\left|C_{k l}^{a} \| C_{k s}^{a *}\right|\right]\left[\sum_{n}\left|C_{n s}^{b}\right|\left|C_{n l}^{b *}\right|\right] \\
\quad \leq \sum_{[s l]}^{\prime} \sqrt{\phi_{l}^{a} \phi_{s}^{a} \phi_{l}^{b} \phi_{s}^{b}} \leq \sqrt{\sum_{[s l]}^{\prime} \phi_{l}^{a} \phi_{s}^{a} \sum_{[s l]}^{\prime} \phi_{l}^{b} \phi_{s}^{b}} .
\end{array}
$$

Working out (38) and combining it with (34) we obtain

$$
\begin{aligned}
\left\|\Theta_{a \neq b}\right\|^{2} & \leq \phi_{a}^{a} \phi_{a}^{b}+\phi_{b}^{a} \phi_{b}^{b} \\
& +\sqrt{\left[1-\left(\phi_{a}^{a}+\phi_{b}^{a}\right)^{2}\right]\left[1-\left(\phi_{a}^{b}+\phi_{b}^{b}\right)^{2}\right]} \equiv F
\end{aligned}
$$

where we used $\sum_{k} \phi_{k}^{r}=1$; see $(35,6)$. We now maximize $F$ in the RHS of (40) so as to obtain a bound on $\left\|\Theta_{a \neq b}\right\|^{2}$ that holds for any $\left\{C_{k l}^{b}\right\}$. The maximization is carried out under two constraints: i) $\phi_{a}^{a} \phi_{b}^{b} \geq|\eta|^{2}$, which follows from applying the Cauchy-Schwartz inequality to (33) with $r=b$ and $p=a$; ii) $\phi_{a}^{a}+\phi_{b}^{a} \leq 1$ and $\phi_{b}^{b}+\phi_{a}^{b} \leq 1$, which follow from the unitarity condition (6). Note from $(39,40)$ that the maximum of $F$ over $\phi_{a}^{b}$ can be reached only at the boundaries of its range, i.e., at $\phi_{a}^{b}=0$ or at $\phi_{a}^{b}=1-\phi_{b}^{b}$. The same holds for $\phi_{b}^{a}$. Direct inspection shows that the maximum of $F$ is reached for $\phi_{a}^{b}=\phi_{b}^{a}=0$ and $\phi_{a}^{a}=\phi_{b}^{b}=|\eta|$ :

$$
\left\|\Theta_{a \neq b}\right\| \leq \sqrt{1-|\eta|^{2}}
$$

Comparing (41) with (27) we see that the maximal amount of the preserved memory on the non-diagonal element is larger for the non-ideal nondiagonal-tonondiagonal transfer than for the diagonal-to-diagonal transfer with the same degree of non-ideality.

For the transfer $\widetilde{r}_{21}=\eta \lambda_{21}$ and for $N=2$ the bound (41) is saturated by the following choice of $\left\{C_{k l}^{b}\right\}$

$$
\begin{aligned}
& C_{11}^{1}=1, \quad C_{21}^{1}=C_{12}^{1}=C_{22}^{1}=0, \\
& C_{21}^{2}=\sqrt{1-|\eta|^{2}}, \quad C_{12}^{2}=\eta, \quad C_{22}^{2}=C_{11}^{2}=0,
\end{aligned}
$$

with an obvious generalization to $N \geq 3$. For the example $(42,43)$ let us write down the final states of $\mathrm{A}(\widetilde{\lambda})$ and $\mathrm{B}(\widetilde{r})$ :

$$
\begin{aligned}
\tilde{\lambda} & =\left(\begin{array}{cc}
\lambda_{11}+\lambda_{22}|\eta|^{2} & \lambda_{12} \sqrt{1-|\eta|^{2}} \\
\lambda_{21} \sqrt{1-|\eta|^{2}} & \lambda_{22}\left(1-|\eta|^{2}\right)
\end{array}\right), \\
\tilde{r} & =\left(\begin{array}{cc}
\lambda_{11}+\lambda_{22}\left(1-|\eta|^{2}\right) & \eta^{*} \lambda_{12} \\
\eta \lambda_{21} & \lambda_{22}|\eta|^{2}
\end{array}\right) .
\end{aligned}
$$

Eq. (44) shows that for a very inaccurate non-diagonalto-non-diagonal transfer $|\eta| \ll 1$, the disturbance introduced in the final state of $\mathrm{A}$ can be a higher-order effect, $\propto|\eta|^{2}$, i.e., in the perturbative sense the disturbance can be neglected. This effect is clearly impossible for the inaccurate diagonal-to-diagonal transfer. There for a small $\varepsilon$ the induced disturbance is at least of order $\varepsilon$; see (26-28). An explanation of this difference is that for the diagonal-to-diagonal transfer the accuracy factor $\varepsilon$ is strictly non-negative. So after the zero-order term 1 in the memory-disturbance factor one can have a first-order term proportional to $\varepsilon$; see (26-28). In contrast, for the non-digonal-to-non-diagonal transfer the accuracy factor $\eta$ is generally complex; thus the first-order factor $|\eta|$ cannot appear (since it is not smooth with respect to $\Re \eta$ and $\Im \eta$ ), and the expansion starts from the second-term $|\eta|^{2}$.

It remains to see what happens to the memory on the diagonal element difference $\lambda_{a a}-\lambda_{b b}$ under non-ideal transfer (31). This memory is quantified by

$$
\frac{1}{\sqrt{2}}\left\|\Theta_{a a}-\Theta_{b b}\right\|,
$$

where the factor $\frac{1}{\sqrt{2}}$ is introduced for convenience. The suitability of this memory measure can be argued for in the same way as after (18). In particular, (46) is equal to its maximal value 1 in the initial state.

We now have:

$$
\left\|\Theta_{a a}-\Theta_{b b}\right\|^{2}=\left\|\Theta_{a a}\right\|^{2}+\left\|\Theta_{b b}\right\|^{2}-2 \operatorname{tr}\left(\Theta_{a a} \Theta_{b b}\right)
$$

Recalling that we are restricted to the c-number situation $\left|C_{k b}^{p}\right\rangle=C_{k b}^{p}|C\rangle$, and denoting $\mu_{l s}^{p r} \equiv \sum_{n} C_{n l}^{p} C_{n s}^{r *}$ we get

$$
\operatorname{tr}\left(\Theta_{a a} \Theta_{b b}\right)=\sum_{l s}\left|\mu_{l s}^{a b}\right|^{2} \geq\left|\mu_{a b}^{a b}\right|^{2}=|\eta|^{2},
$$

where we employed (33) in the last equality. Combining $(47,48)$ with $\left\|\Theta_{a a}\right\| \leq 1$ and $\left\|\Theta_{b b}\right\| \leq 1$, we get

$$
\frac{1}{\sqrt{2}}\left\|\Theta_{a a}-\Theta_{b b}\right\| \leq \sqrt{1-\varepsilon^{2}} .
$$

This is the sought upper bound on the memory of $\lambda_{a a}-$ $\lambda_{b b}$. It has the same form as (41).

For $N=3$ this limit is reached by a unitary in the first three rows of which only the following elements are nonzero

$$
C_{11}^{1}=1, C_{12}^{2}=\varepsilon, C_{32}^{2}=\sqrt{1-\varepsilon^{2}}, C_{13}^{3}=1
$$

This example shows a general property of the nullification of $\left\|\Theta_{a b}\right\|$ in the regime where $\frac{1}{\sqrt{2}}\left\|\Theta_{a a}-\Theta_{b b}\right\|$ is in its maximum (we omit the formal proof of this statement). However, the maximization of $\left\|\Theta_{a b}\right\|$ does not nullify $\frac{1}{\sqrt{2}}\left\|\Theta_{a a}-\Theta_{b b}\right\|$. The example $(42,43)$ illustrates this fact since it leads to $\frac{1}{\sqrt{2}}\left\|\Theta_{a a}-\Theta_{b b}\right\|=1-\varepsilon^{2}$, which is naturally smaller than the optimal bound (49).

\section{A. Extending the bound (41) to more general situations}

In obtaining the bounds $(41,49)$ we constrainted ourselves by (32)-i.e., by one-dimensional Hilbert space $\mathcal{H}_{\mathrm{C}}$, which amounts to a unitary interaction between $\mathrm{A}$ and $\mathrm{B}$ - because so far we were not able to get more general analytic results. It is interesting to know whether taking larger dimensions of $\mathcal{H}_{\mathrm{C}}$ can improve the bounds 
TABLE I: The maximal value of $\left\|\Theta_{a \neq b}\right\|^{2}$ for $\operatorname{dim} \mathcal{H}_{\mathrm{A}}=\mathcal{H}_{\mathrm{B}}=$ 3 and various values of $\operatorname{dim} \mathcal{H}_{\mathrm{C}}$ and the non-ideality parameter $\varepsilon$. The numerical results were obtained via running the NMaximize routine of Mathematica 7 for 37 iterations. The values for $\left\|\Theta_{a \neq b}\right\|^{2}$ are close to the bound (41). For the presented parameters of $\varepsilon$ these bound values are 0.91 and 0.36 .

\begin{tabular}{|c||c|c|}
\hline & $\varepsilon=0.3$ & $\varepsilon=0.8$ \\
\hline \hline $\operatorname{dim} \mathcal{H}_{\mathrm{C}}=2$ & 0.90601 & 0.35990 \\
\hline $\operatorname{dim} \mathcal{H}_{\mathrm{C}}=3$ & 0.90739 & 0.35994 \\
\hline $\operatorname{dim} \mathcal{H}_{\mathrm{C}}=5$ & 0.90997 & 0.35996 \\
\hline
\end{tabular}

TABLE II: The same as in Table I, but for 50 iterations. Convergence to 0.91 and 0.36 [these values are implied by the bound (41)] is seen clearly.

\begin{tabular}{|c||c|c|}
\hline & $\varepsilon=0.3$ & $\varepsilon=0.8$ \\
\hline \hline $\operatorname{dim} \mathcal{H}_{\mathrm{C}}=2$ & 0.90906 & 0.35993 \\
\hline $\operatorname{dim} \mathcal{H}_{\mathrm{C}}=3$ & 0.90913 & 0.35999 \\
\hline $\operatorname{dim} \mathcal{H}_{\mathrm{C}}=5$ & 0.90999 & 0.35999 \\
\hline
\end{tabular}

$(41,49)$. This question was studied numerically for several values of $\operatorname{dim} \mathcal{H}_{\mathrm{C}}$ and $\operatorname{dim} \mathcal{H}_{\mathrm{A}}=\mathcal{H}_{\mathrm{B}}$. We imposed condition (31) and numerically maximized the memories over the available unitary transformations. The standard optimization routine NMaximize of Mathematica 7 has been employed. Our numerical results fully confirmed the bounds $(41,49)$; see Tables I and II. We conjecture that these bounds hold for arbitrary values of $\operatorname{dim} \mathcal{H}_{\mathrm{C}}$.

\section{SUMMARY}

We studied how quantum mechanics constrains the process of transferring density matrix elements from a system A to another system B. It was argued that the problem of matrix elements transfer lies at the core of quantum measurements and quantum state transfer; see section I.

Assuming that the initial density matrix (state) $\lambda$ of A is completely unknown, we show that transferring one diagonal element $\lambda_{a a}$ eliminates the memory on all initial non-diagonal elements $\lambda_{a \neq b}$ from the final state of A.

In contrast, transferring the real part $\Re \lambda_{a \neq b}$ (resp. imaginary part $\Im \lambda_{a \neq b}$ ) of a non-diagonal element $\lambda_{a \neq b}$ eliminates the memory on $\Im \lambda_{a \neq b}$ (resp. $\Re \lambda_{a \neq b}$ ), and in addition the memory on the diagonal element difference $\lambda_{a a}-\lambda_{b b}$ is eliminated. Likewise, transferring $\lambda_{a a}-\lambda_{b b}$ eliminates the memory on both $\Re \lambda_{a \neq b}$ and $\Im \lambda_{a \neq b}$.

Thus there is a complementarity between the diagonal and non-diagonal elements, as well as within the triple $\Re \lambda_{a \neq b}, \Im \lambda_{a \neq b}$ and $\lambda_{a a}-\lambda_{b b}$. Transferring one element of this triple eliminates the memory on two others. Interestingly, transferring one diagonal element implies (in general) more severe consequences for the memory as compared to transferring a difference between two diagonal elements.

We also studied the maximal memory that can be preserved under a finite-accuracy [i.e., non-ideal] transfer. The proper measure of memory is introduced in section VA and shown to posses features necessary for its consistent interpretation. For each type of transfer the maximal memory relates to the amount of non-ideality via system-independent relations. For the transfer of non-diagonal elements we saw that for a very inaccurate transfer, $\varepsilon \ll 1$, the disturbance introduced in the memory can scale as $\varepsilon^{2}$, and thus can be in a sense neglected. This is impossible when transferring diagonal matrix elements.

Below we shall outline relations of our findings with previous results known in literature. Recall that transferring diagonal matrix elements is an essential part of quantum measurement. Our relations-between the accuracy of the diagonal elements transfer and the amount of memory preserved for related non-diagonal elements - resemble uncertainty relations established over the years for characterizing the information obtained during a quantum measurement versus the induced disturbance of the state of the measured system; see [4-9] for recent reviews on this subject.

In the first approach (see, e.g., $[6,8,9]$ ) both the information and disturbance have a global meaning. The information is quantified, e.g., by the Shannon measure $[6,8,9]$, while for characterizing the disturbance one employs the fidelity between the initial and final state of the measured system. The difference with our setup is primarily that we focus on explicitly described quantum measurements and local quantities: the quality of measurement is determined with us by the [relative] accuracy of transferring diagonal matrix element(s). We also use a local measure of memory. Employing here the fidelity (or any other global measure of the state change) will not be adequate. Moreover, as we argue in section V A 1, the fidelity does not posses some features, which are necessary for its consistent application in this problem.

In the second approach the (des)information on the measured variable is quantified via the overall uncertainty of the measured quantity in the Heisenberg representation, while for characterizing the disturbance introduced in the state of the measured system one looks at the statistics of those variables that do not commute with the measured one; see $[4,5]$ for reviews. This approach is well suited for describing the Heisenberg-type uncertainty relations $[4,5]$. Now our approach is more flexible, because it does not insist on doing the full measurement of the system quantity. Indeed, the full measurement would mean transferring all diagonal elements from one system to another. Instead, we concentrate on situations where only some (not all) diagonal elements are transferred. Moreover, our approach studies the transfer of non-diagonal elements that clearly goes beyond the schemes of quantum measurements studied in $[4,5]$. On the other hand, we work in the Schroedinger representation and study disturbances introduced (due to transfer) 
in the memory of the final state of the source system.

With all these differences taken into account, it will be suitable to tell that we presented a new set-up of studying information transfer from one quantum system to another.

\section{Acknowledgements}

It is pleasure to thank R. Balian for discussions.

The work was supported by Volkswagenstiftung.
[1] W. Heisenberg, Physical Principles of Quantum Theory (Dover, NY, 1949).

[2] H. F. Hoffmann, arXiv:quant-ph/0209162.

[3] M. Ozawa, Phys. Rev. Lett. 88, 050402 (2002).

[4] M. Ozawa, Ann. Phys. 311, 350 (2004). Phys. Rev. A 67, 042105 (2003).

[5] P. Busch et al., Phys. Reports, 452, 155 (2007).

[6] L. Maccone, EPL, 77, 40002 (2007).

[7] H. Martens and W. de Muynck, Found. Phys. 20, 255 (1990).

[8] K. Banaszek, Open Syst. Inf. Dyn., 13, 1 (2006); arXiv:0006062 [quant-ph].

[9] F. Buscemi and M. Horodecki, Open Syst. Inf. Dyn., 16, 29 (2009); arXiv:08101310 [quant-ph].

[10] D. Dieks, Phys. Lett. A 92, 271 (1982).

W.K. Wootters and W.H. Zurek, Nature, 299, 802 (1982).

[11] G.M. D'Ariano and H.P. Yuen, Phys. Rev. Lett. 76, 2832 (1996). C.A. Fuchs, Fort. Phys. 46, 535 (1998).

[12] H.P. Yuen, Phys. Lett. A 113, 405 (1986).

[13] T. Mor, Phys. Rev. Lett. 80, 3137 (1998).

[14] R. Jozsa, quant-ph/0204153.

[15] H. Barnum et al., Phys. Rev. Lett. 76, 2818 (1996).

[16] G.M. D'Ariano et al., Phys. Rev. Lett. 95, 060503 (2005).

[17] M. Koashi and N. Imoto, Phys. Rev. A 66, 022318 (2002).

[18] V. Buzek and M. Hillery, Phys. Rev. A 54, 1844 (1996).

[19] L.M. Duan and G.C. Guo, Phys. Rev. Lett. 80, 4999 (1998).

[20] A.K. Pati and S.L. Braunstein, Nature 404, 164 (2000).

[21] P. Deuar and W. J. Munro, Phys. Rev. A 61, 062304 (200).

[22] A. Ferraro et al., J. Phys. A 39, L219 (2006).

[23] V.B. Braginsky and F.Ya. Khalili, Quantum Measurement (Cambridge University, Cambridge, UK, 1992). P. Busch, P. J. Lahti and P. Mittelstaedt, The Quantum Theory of Measurement (Lecture Notes in Physics, Springer, Berlin, 1991).

[24] E. Arthurs and M. S. Goodman, Phys. Rev. Lett. 60, 2447 (1988).

M. Kitano, arXiv:0803.4377 [quant-ph].

[25] J. von Neumann, Mathematical Foundations of Quantum Mechanics (Princeton Univ. Press, Princeton, NJ, 1955). E. P. Wigner, Am. J. Phys., 31, 6 (1963).

[26] A.E. Allahverdyan, R. Balian and Th. M. Nieuwenuizen, Phys. Rev. A 64, 032108 (2001).

[27] F. Haake and D.F. Walls, Phys. Rev. A, 36, 730 (1987). F. Haake and M. Zukowski, Phys. Rev. A, 47, 2506 (1993).

A. Venugopalan, Phys. Rev. A, 61, 012102 (2000). D. Spehner and F. Haake, Phys. Rev. A 77, 052114 (2008).

D. Mozyrsky and V. Privman, Mod. Phys. Letters B, 14,
303 (2000).

W. H. Zurek, Phys. Rev. D 26, 1862 (1982).

A.E. Allahverdyan, R. Balian and Th. M. Nieuwenuizen, Europhys. Lett. 61, 452 (2003).

[28] O.W. Sorensen, Prog. NMR Spectrosc. 21, 503 (1989).

[29] K. Ishikawa et al. Phys. Rev. Lett. 98, 183004 (2007).

[30] A. Abragam and M. Goldman, Rep. Prog. Phys. 41, 395 (1978).

[31] G.D. Scholes, Annu. Rev. Phys. Chem. 54, 57 (2003).

[32] D. Burgarth, Eur. Phys. J. Spec. Top. 151, 147 (2007).

[33] Z. Song and C.P. Sun, Low Temp. Phys. 31, 686 (2005).

[34] L. Viola, E. Knill and R. Laflamme, J. Math. Phys. 34, 7067 (2001).

[35] A.D. Boozer et al., Phys. Rev. Lett. 98, 193601 (2007). H. Kosaka et al., Phys. Rev. Lett. 100, 096602 (2008).

[36] J. R. Taylor, An Introduction to Error Analysis: The Study of Uncertainties in Physical Measurements (University Science Books, 1999).

[37] R. Jozsa, J. Mod. Opt. 41, 2315 (1994).

[38] Consider the differential $\mathrm{d} \widetilde{\lambda}$ of the matrix $\widetilde{\lambda}$ over an infinitesemal increments of two independent real variables $x \equiv \Re \lambda_{a \neq c}$ and $y \equiv \Im \lambda_{a \neq c}[$ see $(7,9)]$ :

$$
\mathrm{d} \tilde{\lambda}=\Theta_{a c}(\mathrm{~d} x+i \mathrm{~d} y)+\Theta_{a c}^{\dagger}(\mathrm{d} x-i \mathrm{~d} y)
$$

To develop the notion of the matrix gradient (similar to the usual gradient) we first calculate the Euclidean norm $\|\mathrm{d} \widetilde{\lambda}\|$ of $\mathrm{d} \widetilde{\lambda}$ :

$$
\begin{aligned}
\|\mathrm{d} \widetilde{\lambda}\|^{2}=\left(\mathrm{d} x^{2}\right. & \left.+\mathrm{d} y^{2}\right)\left[\operatorname{tr}\left(A^{2}\right) n_{x}^{2}\right. \\
& \left.+\operatorname{tr}\left(B^{2}\right) n_{y}^{2}+2 \operatorname{tr}(A B) n_{x} n_{y}\right]
\end{aligned}
$$

where $A \equiv \Theta_{a c}+\Theta_{a c}^{\dagger}, B \equiv i\left(\Theta_{a c}-\Theta_{a c}^{\dagger}\right)$, and where

$$
\left(n_{x}, n_{y}\right)=(\mathrm{d} x, \mathrm{~d} y) / \sqrt{\mathrm{d} x^{2}+\mathrm{d} y^{2}}
$$

is the normal vector over the direction of the small increment. The (modulus of) matrix gradient is defined by maximizing over $\vec{n} \equiv\left(n_{x}, n_{y}\right)$ :

$\frac{1}{4} \max _{\vec{n}}\|\mathrm{~d} \widetilde{\lambda}\|^{2}=\frac{1}{2}\left(\mathrm{~d} x^{2}+\mathrm{d} y^{2}\right)\left[\operatorname{tr}\left(\Theta_{a c} \Theta_{a c}^{\dagger}\right)+\left|\operatorname{tr}\left(\Theta_{a c}^{2}\right)\right|\right]$.

As follows from $\operatorname{tr}(A B) \leq \sqrt{\operatorname{tr}\left(A^{2}\right) \operatorname{tr}\left(B^{2}\right)}$, the quantity

$$
\frac{\max _{\vec{n}}\|\mathrm{~d} \widetilde{\lambda}\|^{2}}{4\left(\mathrm{~d} x^{2}+\mathrm{d} y^{2}\right)}
$$

is limited from above by $\operatorname{tr}\left(\Theta_{a c} \Theta_{a c}^{\dagger}\right)=\left\|\Theta_{a c}\right\|^{2}$, and from below by $\frac{1}{2} \operatorname{tr}\left(\Theta_{a c} \Theta_{a c}^{\dagger}\right)$. Thus, $\left\|\Theta_{a c}\right\|^{2}$ can be taken as an adequate characteristics of the matrix gradient. 